\title{
Review of: "Cross-resistance of cisplatin selected cells to anti-microtubule agents: Role of general survival mechanisms"
}

\author{
Tatiana Massariol Pimenta ${ }^{1}$, Roberto Silva Ribeiro Junior ${ }^{1}$, Bárbara da Silva Martins ${ }^{1}$, Leticia Batista \\ Azevedo Rangel ${ }^{1}$
}

1 Federal University of Espírito Santo

Potential competing interests: The author(s) declared that no potential competing interests exist.

In the current study (PATEL et al., 2021), the impact of anti-microtubule agents was observed in cisplatinsensitive and resistant high-grade serous ovarian cancer cells. A comparison was established between the cisplatin-sensitive cell line used, OVCAR-8, and two cisplatin-resistant ones derived from OVCAR-8,

OVCAR8-CP1 and OVCAR8-CP5. The authors observed that when compared to sensitive cells, OVCAR-CP1 did not show cross-resistance, while OVCAR8-CP5, resistant to a higher cisplatin concentration, showed a subpopulation resistant to anti-microtubule drugs. They suggest that this phenomenon may be related to the pro-survival TNF/NFKB cell signalling pathway. However, the study does not completely elucidate the mechanisms behind cross-resistance. Thus, we highlight some important topics for further discussion about this work.

1. a. As discussed by the authors, OVCAR-8 seems to share histological similarities with high-grade serous ovarian cancer cells. However, it is noteworthy that only a few similarities do not classify OVCAR-8 as HGSOC (Barnes et al., 2021). Thus, this study says little about cross-resistance acquisition to platinumderived compounds and antimicrotubule agents in this subtype. Hence, the use of cell lines known and officially classified as representatives of this subtype, such as OVCAR-3 (Bradbury et al., 2020), OVPA-8 (Tudrej et al., 2018), OVSAHO, OVCAR- 4 and COV318 (Barnes et al., 2021), could provide more reliable conclusions about HGSOC, the most lethal among EOC subtypes.

b. The cell line that was chosen to represent high-grade serous ovarian cancer has characteristics that make it similar to HGSOC (Mitra et al., 2015). Still, we suggest the execution of control experiments performed with an already resistant cell line, such as OVCAR-3, since it has intrinsic resistance to cisplatin (Bradbury et al., 2020). Thus, it would be interesting to consider the use of OVCAR-3, cisplatin-resistant, as a comparative model for OVCAR-8, cisplatin sensitive, to analyze differences and similarities between them.

2. a. In the Materials and methods section, subtopic "Generation of resistant cell lines", it is described that 
resistant cells maintained their resistance for several days, but were not tested for months. One possible interpretation for this sentence is that all experiments were performed during the days the cells were tested for resistance. This statement leads us to understand that this procedure does not mimic the clinical approach very well, since, in patients, the chemotherapy treatment lasts for months and, often, the acquisition of resistance also lasts this amount of time. Thus, the results obtained may extrapolate what the experiments point out.

b. The second possible interpretation for that sentence is that the experiments were carried out in the cells after several months, even without tests to confirm the resistance acquisition and maintenance, previously observed. Thus, the results obtained may not represent a resistance condition.

3. a. In the article, the authors state that it is possible to find the drug concentrations in the figure legends or in the figures themselves. However, it is not possible to find the final concentrations of DMSO used to dilute the antimicrotubule drugs as described in the Materials and methods section. Considering that concentrations above $1 \%$ of DMSO may interfere with the results found, it is important to have access to this information, once DMSO is cytotoxic at higher concentrations (Galvão et al., 2014).

4. a. In the topic "Cisplatin-resistant cell lines do not show increased activity of either ABCB1 or ABCG2", the authors stated that there is no expression of $A B C B 1$ and ABCG2 receptors in cisplatin-resistant cells, leaving it unclear whether it is a protein or mRNA expression. However, in the topic "Gene set enrichment analysis (GSEA) reveals enrichment for survival pathways within a subpopulation of OVCAR8-CP5 cells", overexpression of mRNA of these two transporters in only one subpopulation was demonstrated, which does not represent the heterogeneous tumour mass. Since it does not specify the analyzed target, protein or mRNA, the affirmation in the first topic makes these two pieces of information contradictory and confusing, thus decreasing the impact of these experiments when taken together.

b. In the Materials and methods section, it was not clear the reason for analyzing the subpopulation that was determined or the reason for reanalyzing a subpopulation of a cell line that had already been tested which demonstrated a negative result. Noteworthy, it was not specified by which method the subpopulation was isolated and analyzed, as well as which markers were present in this subpopulation.

The discussion about these topics is crucial, as it enriches the current work and makes it even more relevant to the scientific community. Thus, the clarification of these points avoids extrapolating the biological meaning of the results and, consequently, future inquiries about.

\section{REFERENCES:}


Mitra AK, Davis DA, Tomar S, Roy L, Gurler H, Xie J, Lantvit DD, Cardenas H, Fang F, Liu Y, Loughran E, Yang J, Sharon Stack M, Emerson RE, Cowden Dahl KD, V Barbolina M, Nephew KP, Matei D, Burdette JE. In vivo tumor growth of high-grade serous ovarian cancer cell lines. Gynecol Oncol. 2015 Aug;138(2):372-7. doi: 10.1016/j.ygyno.2015.05.040. Epub 2015 Jun 5. PMID: 26050922; PMCID: PMC4528621.

Bradbury A, O'Donnell R, Drew Y, Curtin NJ, Sharma Saha S. Characterization of Ovarian Cancer Cell Line NIH-OVCAR3 and Implications of Genomic, Transcriptomic, Proteomic and Functional DNA Damage Response Biomarkers for Therapeutic Targeting. Câncer (Basel) . 2020; 12 (7): 1939. Published 2020 Jul 17. Doi: 10.3390 / cancers12071939

Barnes BM, Nelson L, Tighe A, et al. Distinct transcriptional programs stratify ovarian cancer cell lines into the five major histological subtypes. Genome Med. 2021;13(1):140. Published 2021 Sep 1.

doi:10.1186/s13073-021-00952-5

Galvao J, Davis B, Tilley M, Normando E, Duchen MR, Cordeiro MF. Unexpected low-dose toxicity of the universal solvent DMSO. FASEB J. 2014 Mar;28(3):1317-30. doi: 10.1096/fj.13-235440. Epub 2013 Dec 10. PMID: 24327606. 